\title{
Model characterization and mechanical property analysis of bimetallic functionally graded turbine discs
}

\author{
Yiwei Dong ${ }^{1,2,}{ }^{*}$, Weiguo Yan $^{1}$, Tao Liao ${ }^{1}$, Qianwen Ye ${ }^{1}$, and Yancheng You ${ }^{1,2}$ \\ ${ }^{1}$ School of Aerospace Engineering, Xiamen University, 422 South Siming Road, Xiamen, 361005, People's Republic of China \\ 2 Shenzhen Research Institute, Xiamen University, No. 19, Gaoxin South 4th Road, Nanshan District, Shenzhen, 518057, \\ People's Republic of China
}

Received: 23 September 2020 / Accepted: 6 January 2021

\begin{abstract}
In advanced propulsive systems, a turbine disc bears vast mechanical and thermal loads under its working conditions of high-temperature gradients and high rotational velocity. The complex working conditions of aero-engine turbine discs place stringent performance requirements on the materials used. With dual organizations and superior composite performances, bimetallic functionally graded turbine discs have become a focus in the research of high thrust-to-weight ratio aero-engines. To study the mechanical properties of new bimetallic functionally graded materials under service conditions, we propose a volumetric fraction expression and adjustable composition distribution parameters that are suitable for simulating the composition distribution of bimetallic functionally graded turbine discs. On this basis, a characterization model for functionally graded materials based on the analysis of the internal thermodynamic properties of bimetallic turbine discs is established. The thermodynamic properties and fatigue performances of functionally graded materials under service conditions are analysed. Mechanical property simulations of functionally graded turbine discs are performed using different composition distribution parameters, and reasonable ranges are determined for the various composition distribution parameters. The results show that bimetallic functionally graded turbine discs are suitable for high-stress-gradient and high-temperaturegradient environments with lower weights than those of current GH4169 alloy turbine discs.
\end{abstract}

Keywords: Functionally graded material / Turbine disc / Finite element analysis / Mechanical property / Low-cycle fatigue

\section{Introduction}

As key components of aero-engines, high-pressure turbine discs must operate in harsh high-temperature and highrotational-speed environments over long periods of time. The reliability of such complex thermal mechanical machinery has a direct effect on flight safety. The extreme and unusual operating conditions and operational requirements of turbine discs pose enormous challenges on material and manufacturing technology innovation. The emergence of various new materials, such as composite materials, multiphase materials, and functionally gradient materials, and the development and advancement of additive material manufacturing technology have made materials designable in terms of strength, stiffness, and service reliability.

The working conditions of aero-engine turbine discs are extremely harsh. The rim of the disc, being exposed to hightemperature gas at operating temperatures of $450-840^{\circ} \mathrm{C}$,

\footnotetext{
* e-mail: yiweidong@xmu.edu.cn
}

must possess superior durability and resistance against creep and fatigue cracking. Conversely, the center of the disc operates at relatively low temperatures but is subject to large centrifugal stresses, so the center must have a high yield strength and good low-cycle fatigue performance [1]. These characteristics cannot be satisfied by a single material $[2-5]$. Functionally gradient materials (FGMs), on the other hand, have the distinct advantage that their properties can vary with the gradient and the location on the part [6], and thus, they can withstand the complex operational environments and satisfy the requirements of turbine discs. For a bimetallic functionally graded turbine disc, the structural design can be fully optimized, and the stability of the structure can be ensured without increasing the thickness or weight of the structure.

The ultimate purpose of establishing a thermophysical model of functionally gradient materials is to determine the optimum material volume fraction formula so that the material can exhibit the best thermophysical properties in the service process. That is, the interface of the component material is blurred, and the concentrated thermal stress 


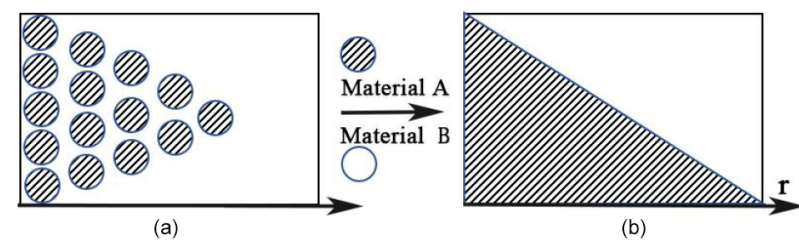

Fig. 1. Idealized functionally graded material.

is relieved. Figure 1a shows a schematic diagram of a functionally graded material composed of metallic materials $\mathrm{A}$ and $\mathrm{B}$. As the radius $\mathrm{r}$ increases, the volume fraction of material A gradually decreases and the volume fraction of material B gradually increases. Ignoring the shapes of both materials and the contact problem, the composition distribution of the functionally graded material may be simplified to that of an idealized FGM [7], as shown in Figure 1b. As the gradient of the volume fraction changes, the thermomechanical properties of the material, such as the modulus of elasticity, Poisson's ratio, coefficient of linear expansion, and thermal conductivity, will also produce gradient changes.

The finite element method is widely used in the analysis of thermal stress in functionally graded materials. In-depth studies of the thermal responses of functionally graded materials under different conditions [8,9] have been conducted by a number of investigators. In numerical simulations of functionally graded materials, some researchers [10,11] used the ANSYS finite element software for the numerical simulation of FGMs and effectively proved that FGMs significantly improve the mitigation of the thermal stress and the suppression of interfacial crack growth. They have also verified the reliability of finite element software in the auxiliary design of FGMs, but some flaws still remain in the continuity features for the transition between the layers of the numerical simulations of FGMs.

Other finite element related studies include the thermal stress calculation formula for thin FGM sheets under thermal loads derived by Jiang [12]. With the aid of secondary development in ABAQUS, the steady-state thermal problem of a one-dimensional FGM thin plate was analyzed on the basis of classical plate theory. Pourhamid et al. [13] introduced the super element method (SEM) and analyzed the metal-ceramic FGM engine piston cylinder problem in ABAQUS and examined the effect of the material composition distribution on the distribution of the temperature and stress fields.

In this work, based on a combination of theoretical analysis and finite element simulations, we derived and calculated the thermodynamic parameters of FGMs for different composition ratios, conducted numerical simulations of FGMs under service conditions for different composition ratios, and performed an optimization analysis of the composition distribution parameters based on the simulation results. We established a material characterization model for FGMs aimed at tackling the thermodynamic problem of a bimetallic turbine disc and built a set of material property parameters for FGMs that was suitable for turbine disc service conditions with good thermodynamic performances.

\section{Bimetallic functionally graded material model characteristics}

\subsection{Composition distribution function and material selection of functionally graded materials}

Some scholars have achieved fruitful results in the study of the composition distribution formula of functionally graded materials and related mechanical problems, but some methods are computationally intensive and timeconsuming, and the complete power function form has the problem of difficult data processing. To establish a simplified and practical model that can be applied quickly to actual engineering problems, in this paper, we adopt a simplified power function as the expression for the volume fraction of the material:

$$
f_{\alpha}(r)=C_{0} \cdot\left[1-k \cdot\left(\frac{r}{B}\right)^{n}\right]
$$

where $\alpha$ represents the A phase material, $\mathrm{C}_{0}, k$, and $n$ are adjustable coefficients that control the shape of the curve (hereinafter referred to as composition distribution parameters), and $\mathrm{B}$ is the maximum radius of the turbine disc.

The working conditions of aero-engine turbine discs are extremely harsh. The rim of the disc, being exposed to hightemperature gas at operating temperatures of $450-840^{\circ} \mathrm{C}$, must possess superior durability and resistance against creep and fatigue cracking. Conversely, the center of the disc operates at relatively low temperatures but is subject to large centrifugal stresses, so the center must have a high yield strength and good low-cycle fatigue performance. Below $650^{\circ} \mathrm{C}$, the yield strength of GH4169 ranks the highest among wrought superalloys, and it has good resistance against fatigue, radiation, oxidation, and corrosion. This makes it a suitable hub material. Below $900^{\circ} \mathrm{C}$, the K418B alloy has high resistance against creep, thermal fatigue, and oxidation, which makes it a suitable rib material.Because the superalloys GH4169 and K418B are commonly used turbine disc materials, we examine bimetallic functionally graded materials based on these two materials. The material parameters of the GH4169 and K418B alloys are affected by the working temperature. The specific thermal parameters are shown in Tables 1 and 2, respectively. Based on the properties of the two materials, we use the GH4169 alloy as the $\alpha$ phase material in this paper, with its volume fraction decreasing in the radial direction, and the K418B alloy as the $\beta$ phase material, with its volume fraction increasing in the radial direction.

\subsection{Thermodynamic parameters of functionally graded materials}

When the volume fraction $f_{\alpha}(r)$ of the $\alpha$ phase material is known, the change of the elastic mechanical parameters of the FGM with radius $r$ can be expressed as follows [15]:

$$
\begin{gathered}
E(r)=f_{\alpha}(r) \cdot E_{1}+\left[1-f_{\alpha}(r)\right] \cdot E_{2}, \\
\nu(r)=f_{\alpha}(r) \cdot v_{1}+\left[1-f_{\alpha}(r)\right] \cdot v_{2} .
\end{gathered}
$$


Table 1. Temperature dependence of thermodynamic parameters of GH4169 superalloy [14].

\begin{tabular}{lllllll}
\hline $\begin{array}{l}\text { Temperature } \\
\left({ }^{\circ} \mathrm{C}\right)\end{array}$ & $\begin{array}{l}\text { Elastic } \\
\text { modulus E } \\
(\mathrm{GPa})\end{array}$ & $\begin{array}{l}\text { Poisson's } \\
\text { ratio } v\end{array}$ & $\begin{array}{l}\text { Coefficient } \\
\text { of linear } \\
\text { expansion } \\
\alpha\left(10^{-6} \mathrm{C}^{-1}\right)\end{array}$ & $\begin{array}{l}\text { Thermal } \\
\text { Conductivity } \\
\lambda\left(W /\left(m \cdot{ }^{\circ} \mathrm{C}\right)\right)\end{array}$ & $\begin{array}{l}\text { Specific } \\
\text { heat } \\
\mathrm{c}\left(\mathrm{J} /\left(\mathrm{kg} \cdot{ }^{\circ} \mathrm{C}\right)\right)\end{array}$ & $\begin{array}{l}\text { Density } \\
\rho\left(\mathrm{kg} / \mathrm{m}^{3}\right)\end{array}$ \\
\hline 20 & 205.0 & 0.321 & 11.8 & 13.40 & $\backslash$ \\
300 & 184.5 & 0.329 & 13.5 & 17.59 & 481.4 \\
400 & 175.5 & 0.339 & 14.1 & 18.84 & 493.9 & 8240 \\
500 & 168.5 & 0.344 & 14.4 & 20.10 & 539.0 & 556.2 \\
600 & 153.0 & 0.355 & 14.8 & 21.77 & 594.7 \\
650 & 142.0 & 0.361 & 15.1 & 22.30 & 23.63 & \\
750 & 130.5 & 0.381 & 16.2 & 230.8 & \\
\hline
\end{tabular}

Table 2. Temperature dependence of thermodynamic parameters of K418B superalloy [14].

\begin{tabular}{lllllll}
\hline $\begin{array}{l}\text { Temperature } \\
\left({ }^{\circ} \mathrm{C}\right)\end{array}$ & $\begin{array}{l}\text { Elastic } \\
\text { modulus } \\
\mathrm{E}(\mathrm{GPa})\end{array}$ & $\begin{array}{l}\text { Poisson's } \\
\text { ratio } v\end{array}$ & $\begin{array}{l}\text { Coefficient } \\
\text { of linear } \\
\text { expansion } \\
\alpha\left(10^{-6}{ }^{\circ} \mathrm{C}^{-1}\right)\end{array}$ & $\begin{array}{l}\text { Thermal } \\
\text { Conductivity } \\
\lambda\left(W /\left(\mathrm{m} \cdot{ }^{\circ} \mathrm{C}\right)\right)\end{array}$ & $\begin{array}{l}\text { Specific } \\
\text { heat } \\
\mathrm{c}\left(\mathrm{J} /\left(\mathrm{kg} \cdot{ }^{\circ} \mathrm{C}\right)\right)\end{array}$ & $\begin{array}{l}\text { Density } \\
\rho\left(\mathrm{kg} / \mathrm{m}^{3}\right)\end{array}$ \\
\hline 10 & 211 & 0.29 & $\backslash$ & $\backslash$ & $\backslash$ \\
300 & 195 & 0.28 & 12.9 & 12.98 & 481 \\
400 & 190 & 0.29 & 12.9 & 14.65 & 490 & 8080 \\
500 & 184 & 0.30 & 13.4 & 16.33 & 515 & \\
600 & 179 & 0.29 & 13.7 & 18.42 & 544 & \\
700 & 171 & 0.31 & 14.2 & 20.52 & 559 \\
800 & 165 & 0.30 & 14.7 & 22.61 & 592 \\
\hline
\end{tabular}

Based on the knowledge of the elastic mechanics, the expressions of the bulk modulus $K(r)$, shear modulus $G(r)$, and Lame constant $\lambda(r)$ are as follows:

$$
\begin{gathered}
K(r)=\frac{E(r)}{1-2 v(r)} \\
G(r)=\frac{E(r)}{2[1+v(r)]} \\
\lambda(r)=\frac{K(r)-2 G(r)}{3}=\frac{E(r) v(r)}{[1-2 v(r)][1+v(r)]}
\end{gathered}
$$

When the volume fraction $f_{\alpha}(r)$ of the $\alpha$ phase material is known, the change of the thermodynamic parameters of the FGM with the radius $r$ can be expressed as follows [16]:

$$
\begin{gathered}
\alpha(r)=f_{\alpha}(r) \cdot \alpha_{1}+\left[1-f_{\alpha}(r)\right] \cdot \alpha_{2} \\
k(r)=\frac{k_{1} k_{2}}{k_{1} \cdot\left[1-f_{\alpha}(r)\right]+k_{2} \cdot f_{\alpha}(r)}
\end{gathered}
$$

After determining the functional relationship between the parameters of the functionally graded material and the volume fraction of the $\alpha$ phase material, the performance parameters are correlated with the radius $r$ of the turbine disc, forming a theoretical basis for establishing the functionally graded material model in the finite element software and calculating its service performance.

\subsection{Constitutive model of turbine disc}

In view of the large dynamic loads of the high-speed airflow impact and burst experienced by turbine discs in service, the Johnson-Cook (J-C) empirical model [17] is often used in the simulation of the mechanical properties of turbine discs in actual operating conditions. The model is widely used to describe the strength limits and failure behaviors of metallic materials in high-temperature environments.

Since some of the parameters of the J-C model must be obtained through experiments, including quasi-static tensile testing and dynamic tensile and compression testing and the functionally graded bimetallic materials studied herein are still in the theoretical validation stage, experimental data are not available. If we establish a theoretical J-C model based on an unverified bimetallic FGM, it is extremely likely to contain large errors. Given that there have been successful J-C studies worldwide for the GH4169 alloy, we adopted the experimental data of 
Table 3. GH4169 alloy Johnson-Cook (J-C) model parameters.

\begin{tabular}{lll}
\hline $\begin{array}{l}\text { Material } \\
\text { parameters }\end{array}$ & Physical meaning & Value \\
\hline $\mathrm{A}$ & Initial yield stress (MPa) & 650 \\
$\mathrm{~B}$ & Hardening constant (MPa) & 809.16 \\
$\mathrm{C}$ & Strain rate constant & -0.0067 \\
$\mathrm{n}$ & Hardening Index & 0.2754 \\
$\mathrm{~m}$ & Thermal softening index & 1.534 \\
\hline
\end{tabular}

the GH4169 alloy [18] to build the constitutive model and minimize the error of the J-C model. follows:

The mathematical expression of the $\mathrm{J}-\mathrm{C}$ model is as

$$
\begin{gathered}
\sigma_{e}=\left(A+B \varepsilon_{e}^{n}\right)\left(1+C \ln \dot{\varepsilon}_{e}^{*}\right)\left(1-T^{*} m\right) \\
T^{*}=\frac{T-T_{r}}{T_{m}-T_{r}} \\
\dot{\varepsilon}_{e}^{*}=\frac{\dot{\varepsilon}_{p}}{\dot{\varepsilon}_{0}}
\end{gathered}
$$

where $A, B, n, C$, and $m$ are parameters determined by the material, $\sigma e$ is the von Mises equivalent flow stress, $\varepsilon_{\mathrm{e}}$ is the equivalent plastic strain, $\dot{\varepsilon}_{e}^{*}$ is the dimensionless strain rate, $\dot{\varepsilon}_{p}$ is the equivalent plastic strain rate, $\dot{\varepsilon}_{0}$ is the reference strain rate, taken to be $0.001 \mathrm{~s}-1, T^{*}$ is the dimensionless temperature, and $T, T_{r}$, and Tm are the test temperature, room temperature $\left(20^{\circ} \mathrm{C}\right)$, and melting point, respectively. The material parameters obtained after fitting the J-C model are shown in Table 3.

\subsection{FGM turbine disc performance calculation program}

In this study, we developed a calculation program for the thermodynamic responses of bimetallic functionally graded materials. The concepts of the calculation are shown in Figure 2. The program performs model import, material setting, and mesh division. The interface is shown in Figure 3 .

\section{Mechanical performance analysis of bimetallic functionally graded turbine disc}

\subsection{Model establishment for turbine disc}

Considering that the main goal of this research is to explore the influence of functionally graded materials on the service performances of turbine discs, a simplified model of a certain type of aero-engine turbine disc was selected for the modeling. Due to the symmetry of the structure and working conditions of the aero-engine turbine disc, a quarter scale of the turbine disc was used for the

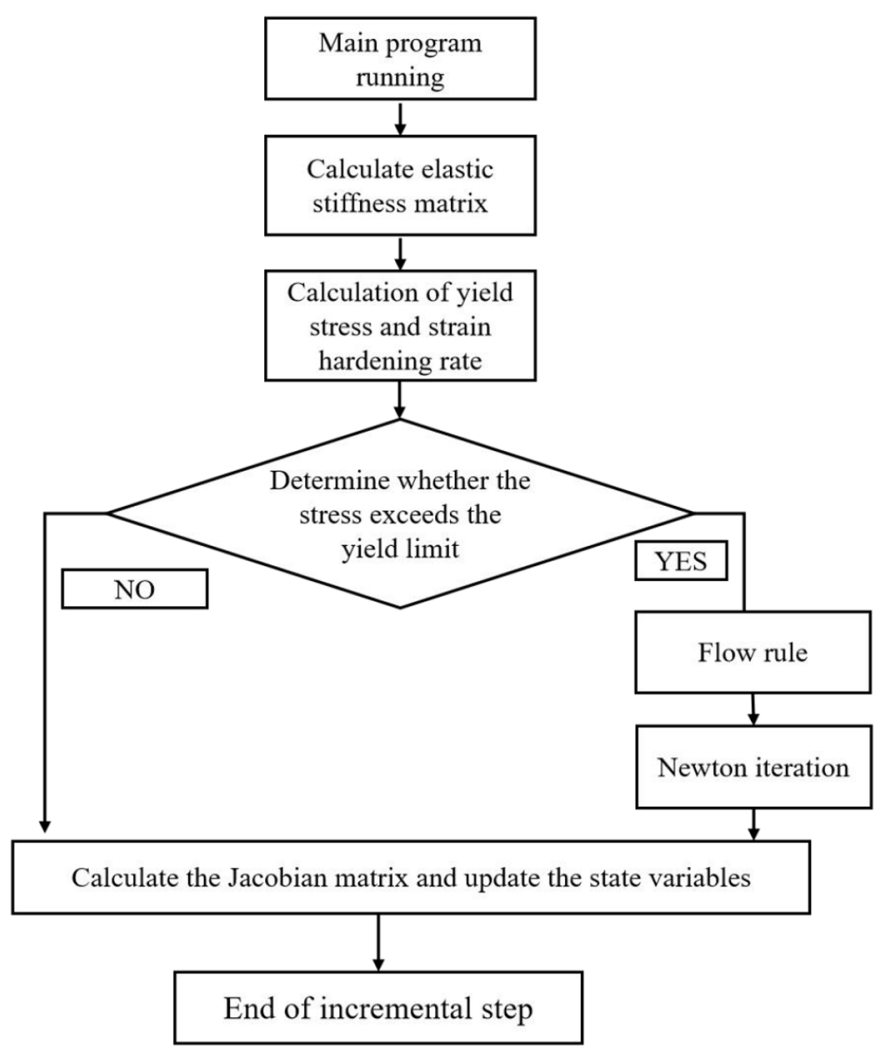

Fig. 2. Operating process of the calculation program.

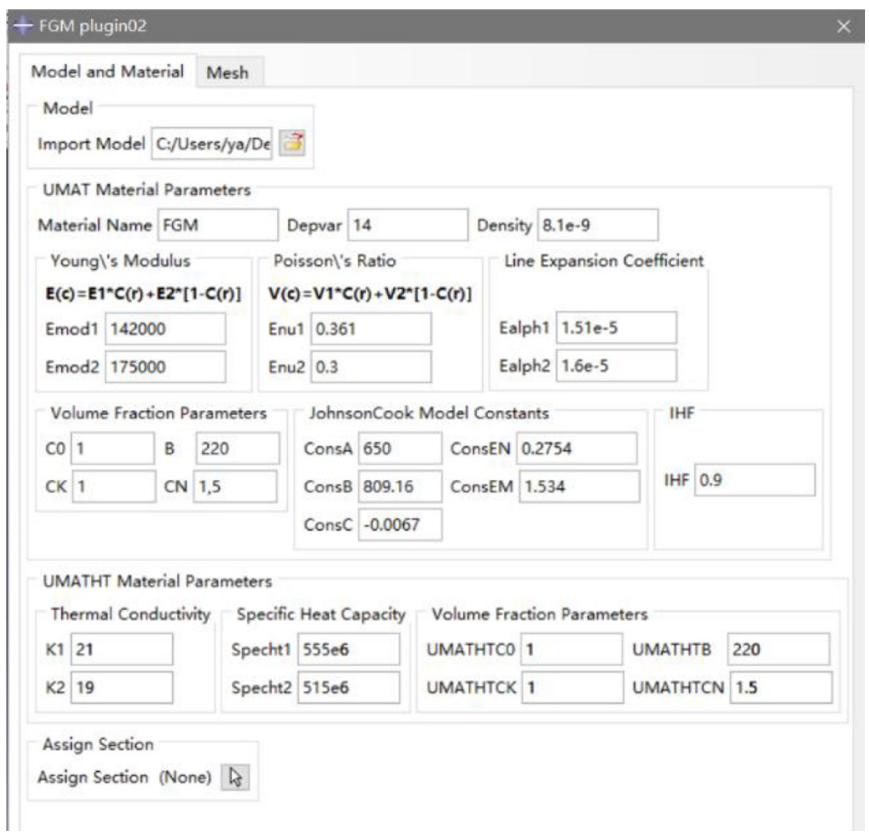

Fig. 3. Setting interface of functionally graded material (FGM) properties.

simulations to improve the calculation efficiency. The turbine disc model and the material distribution are shown in Figure 4. 


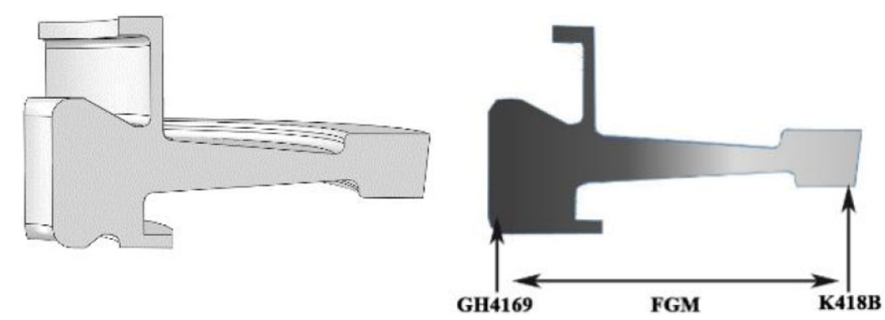

Fig. 4. Turbine disk model and material distribution.

Table 4. Load spectrum of turbine disc at $750 \mathrm{~h} \mathrm{[21].}$

\begin{tabular}{lll}
\hline Working condition & $\begin{array}{l}\text { Turbine disc speed } \\
(\mathrm{rpm})\end{array}$ & $\begin{array}{l}\text { Number } \\
\text { of cycles }\end{array}$ \\
\hline Start-max-start & $0-17650-0$ & 1280 \\
Idle-Max-Idle & $9210-17650-9210$ & 1845 \\
Cruise-max-cruise & $16446-17650-16446$ & 23300 \\
\hline
\end{tabular}

Table 5. Centrifugal load on blade corresponding to various speeds of turbine disc.

\begin{tabular}{lll}
\hline Working condition & Speed $(\mathrm{rpm})$ & Pressure $(\mathrm{MPa})$ \\
\hline Maximum state & 17650 & 200 \\
Idle state & 9210 & 68 \\
Cruise state & 16446 & 184 \\
\hline
\end{tabular}

\subsection{Boundary conditions and load settings of turbine disc}

A rotating hot disc in a turbine engine is in general subjected to a combination of centrifugal loads, tensile stress equivalent to the centrifugal force generated by the blade, aerodynamic force generated by the impact of the oncoming airflow on the blade, thermal stress, vibration load and assembly stress. Among them, the centrifugalload and thermal stress have a greater impact on the performance of the turbine disc, while the other three types of loads have a lower impact. Therefore, the load analysis of the turbine disc in this paper mainly includes the centrifugal load and the temperature load [19].

To simulate the temperature field under real service conditions, a sequential coupling method is adopted for the finite element thermal stress analysis. It is assumed that the temperature field is in a steady state, the temperature of the core is $360^{\circ} \mathrm{C}$, and the temperature of the rim is $600{ }^{\circ} \mathrm{C}[20]$.

The centrifugal load on the turbine disc can be regarded as a superposition of the centrifugal force of the disc itself and the tensile force on the disc by the centrifugal force of the blades. The spectrum of the rotational load of the disc is shown in Table 4, and the parameters of the applied centrifugal load are shown in Table 5.
Table 6. Stress, strain, and total deformation of FGM turbine disc under different engine conditions.

\begin{tabular}{lllll}
\hline $\begin{array}{l}\text { Engine } \\
\text { state }\end{array}$ & $\begin{array}{l}\text { Speed } \\
(\mathrm{rpm})\end{array}$ & $\begin{array}{l}\text { Max von } \\
\text { Mises } \\
\text { stress } \\
(\mathrm{MPa})\end{array}$ & $\begin{array}{l}\text { Maximum } \\
\text { principal } \\
\text { strain }\end{array}$ & $\begin{array}{l}\text { Total } \\
\text { deformation } \\
(\mathrm{mm})\end{array}$ \\
\hline $\begin{array}{l}\text { Maximum } \\
\text { state }\end{array}$ & 17650 & 914.3 & $6.625 \mathrm{e}-3$ & 0.4269 \\
$\begin{array}{l}\text { Cruise state } \\
\text { Idle state }\end{array}$ & 16446 & 808.3 & $5.860 \mathrm{e}-3$ & 0.3786 \\
\hline
\end{tabular}

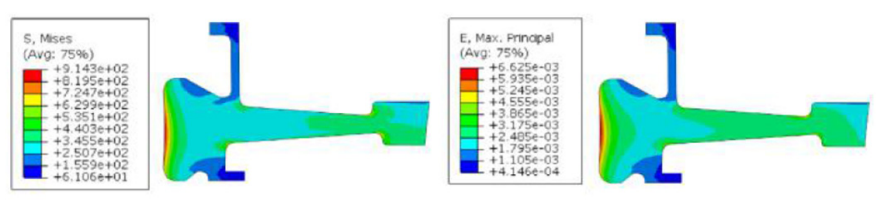

(a)

(b)

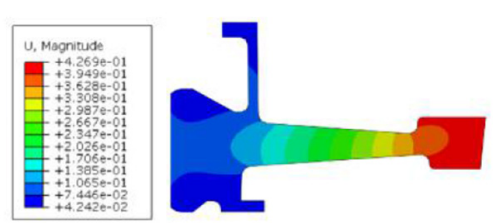

(c)

Fig. 5. Simulation results of FGM turbine disk in maximum state: (a) von Mises stress distribution nephogram, (b) strain distribution nephogram, and (c) deformation distribution nephogram.

\subsection{Stress-strain analysis of turbine disc}

The simulation results of the turbine disc with the engine speed in the maximum state, idle state, and cruise state are presented in this section. The stress, strain, and deformation of the FGM turbine disc under different engine conditions are shown in Table 6.

As shown in Figures 5-7, the von Mises stress distribution of the turbine disc in the various states showed that the stress decreased as the radius increased. The maximum stress was at the hub of the disc, so the hub portion of the bimetallic functionally graded turbine disc was the location with the risk of failure. Furthermore, as the speed of the turbine disc increased, the magnitudes of the stress, strain, and total deformation all increased.

To study the performance difference between the functionally graded bimetallic turbine disc and the GH4169 alloy turbine disc, we chose the simulation results of a GH4169 alloy disc operating in the maximum state of the engine and under the same load and boundary conditions as those of the FGM disc, as shown in Figure 8. The performance comparison between the two is shown in Table 7.

The above results show that the location with the maximum von Mises stress was at the hub for both the FGM disc 


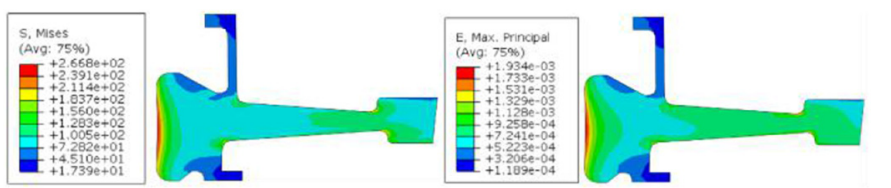

(a)

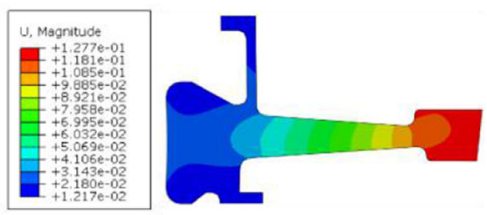

(c)

Fig. 6. Simulation results of FGM turbine disk in idle state: (a) von Mises stress distribution nephogram, (b) strain distribution nephogram, and (c) deformation distribution nephogram.
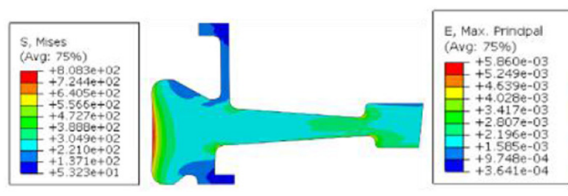

(a)

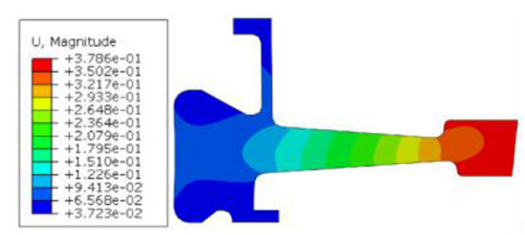

(c)

Fig. 7. Simulation results of FGM turbine disc in cruising state: (a) von Mises stress distribution nephogram, (b) strain distribution nephogram, and (c) deformation distribution nephogram.
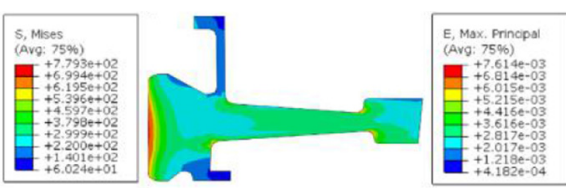

(a)

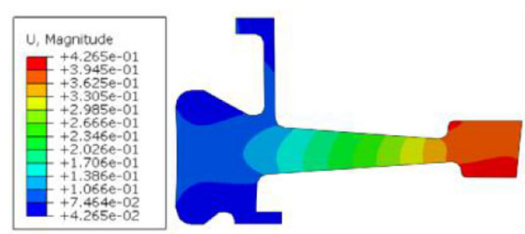

(c)

Fig. 8. Simulation results of GH4169 alloy turbine disc in maximum state: (a) von Mises stress distribution nephogram, (b) strain distribution nephogram, and (c) deformation distribution nephogram. (b)

Table 7. Comparison of performance between FGM turbine disc and GH4169 disc in maximum state.

\begin{tabular}{llll}
\hline Material & $\begin{array}{l}\text { Max von } \\
\text { Mises stress } \\
(\mathrm{MPa})\end{array}$ & $\begin{array}{l}\text { Maximum } \\
\text { principal } \\
\text { strain }\end{array}$ & $\begin{array}{l}\text { Total } \\
\text { deformation } \\
(\mathrm{mm})\end{array}$ \\
\hline FGM & 914.3 & $6.625 \mathrm{e}-3$ & 0.4269 \\
GH4169 & 779.3 & $7.614 \mathrm{e}-3$ & 0.4265 \\
\hline
\end{tabular}

Table 8. Values of FGM fatigue parameters.
Fatigue parameter

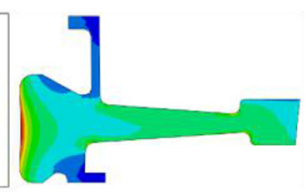

(b)

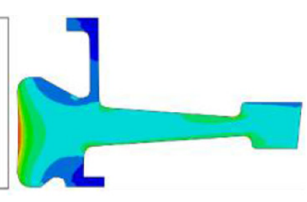

(b) and the GH4169 disc. The high-risk positions of the two material discs were the same, but the maximum von Mises stress of the FGM disc was greater than that of the GH4169 disc. The maximum principal strain for the two material turbine discs differed very little, and the magnitude and distribution of the total deformation on the turbine discs of the two different materials were basically the same, with the maximum total deformation on both at the rim.

\subsection{Fatigue life analysis of turbine disc}

Based on the analysis results above, the fatigue life of the center hole of the turbine disc was studied. The FGM fatigue parameters are shown in Table 8, where $\alpha$ is the elongation of the material. Combined with the loading conditions of aero-engine turbine discs in actual service, the turbine disc load spectrum used in this study is shown in Table 9. 


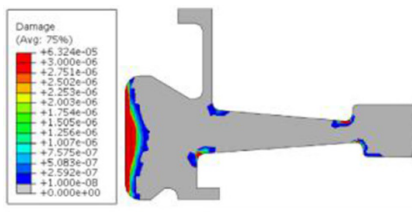

(a)

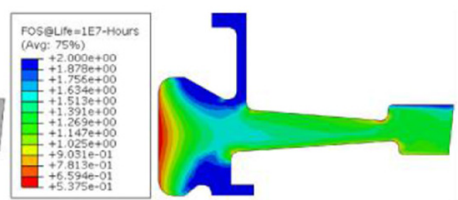

(b)

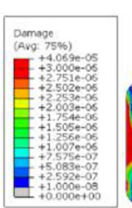

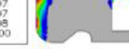

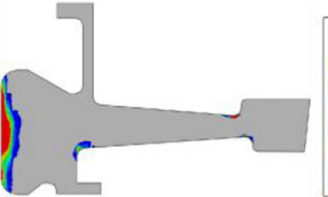

(a)

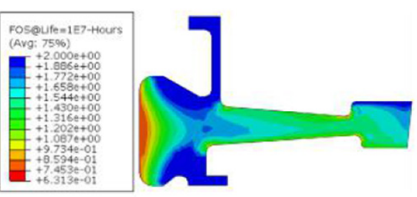

(b)

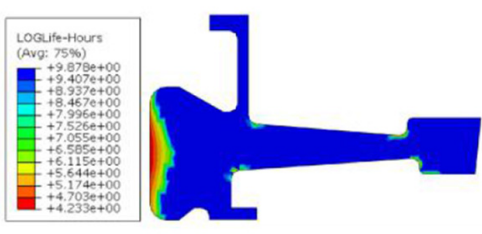

(c)

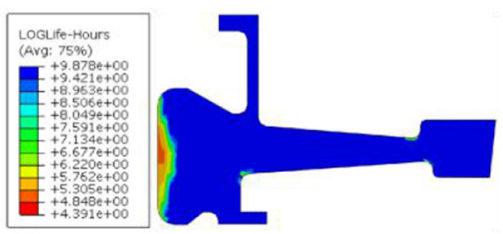

(c)

Fig. 9. Results of fatigue damage, FOS factor, and logarithmic fatigue life of FGM disc at maximum speed: (a) fatigue damage distribution of FGM disc, (b) FOS factor distribution nephogram of FGM disc, and (c) logarithmic fatigue life distribution nephogram of FGM disc.

According to the engine performance simulation requirements, the simulated upper limit speed will exceed the maximum speed, and the lower limit of the rotational speed was taken to be $5 \%$ of the highest simulated speed [22]. When choosing the fatigue algorithm, in view of the ductile mechanical properties of the bimetallic material, we chose to adopt the multi-axial fatigue analysis method within the scope of the nominal stress method.

The fatigue analysis in this section mainly considers the fatigue performance of the turbine disc in the maximum state of the engine. The fatigue damage in the maximum state is regarded as the most severe fatigue damage in the service process, and the fatigue life in the maximum state is regarded as the shortest fatigue life in the service process.

Figure 9 shows the results of fatigue damage (henceforth referred to as damage), factor of strength (FOS), and fatigue life (henceforth referred to as life) of the FGM turbine disc at the maximum speed using the initial composition distribution parameters. As shown in Figure 9a, the fatigue damage of the FGM disc was mainly distributed at the center hole and sharp corners of the turbine disc, that is, the high-risk area in the stress analysis. The largest damage was $6.324 \times 10^{-5}$, which appeared at the center hole of the disc.

When selecting a fatigue-resistant structural design method in the FE-SAFE software package (Dassault Systèmes, France), we chose the FOS factor design method and took the default design life to be $10^{6} \mathrm{~h}$. In the FESAFE, the calculated life and the design life were compared during the calculation. If the calculated life was shorter than the design life, the FOS factor of the node was less than 1; if the calculated life was longer than the design life, the FOS factor of the node was greater than 1. Figure 9b shows that the area where the FOS factor was less than 1 was the center hole of the disc, indicating that the fatigue life at this location was less than the design life of $10^{6} \mathrm{~h}$. The minimum value of the FOS factor of the FGM disc was 0.5375. As shown in Figure 9c, the shortest life of the FGM
Table 10. Comparison of turbine disc fatigue characteristics for two materials in maximum state.

\begin{tabular}{llll}
\hline Material & $\begin{array}{l}\text { Maximum } \\
\text { fatigue } \\
\text { damage }\end{array}$ & $\begin{array}{l}\text { Minimum } \\
\text { FOS factor }\end{array}$ & $\begin{array}{l}\text { Shortest } \\
\text { fatigue life } \\
\text { (hours) }\end{array}$ \\
\hline FGM & $6.324 \mathrm{e}-5$ & 0.5375 & 17100.15 \\
GH4169 & $4.069 \mathrm{e}-5$ & 0.6313 & 24603.68 \\
\hline
\end{tabular}

disc, $17100.15 \mathrm{~h}$ or approximately 20384 cycles, occurred at the center hole of the FGM disc, which satisfied the low cycle fatigue life interval of $10^{4}-10^{5} \mathrm{~h}$.

We performed fatigue analysis for the GH4169 alloy turbine disc operating under the same conditions, and the analysis results of the fatigue damage, FOS factor, and logarithmic fatigue life for maximum rotational speed are shown in Figure 10.

The results showed that the locations of fatigue damage were the same for the GH4169 alloy disc and FGM disc. The location for the shortest fatigue life occurred at the center hole, which agreed with the predicted high-risk location in the stress analysis and with the shortest life on the FGM disc. The radial distribution of the FOS factor differed significantly from that of the FGM disc, but the location for the minimum FOS factor was the same as that on the FGM disc. Table 10 shows a comparison of the fatigue behaviors of the two materials.

Based on the above analysis results, the fatigue performance of the FGM disc with the initial composition distribution parameters was poorer than that of the GH4169 disc in the maximum state. The shortening of the fatigue life at the high-risk zone of the central hole on the disc was particularly significant, but the FGM disc still conformed to the low-cycle fatigue law of aero-engine turbine discs, and its fatigue life was within the acceptable range of the low-cycle fatigue life. 


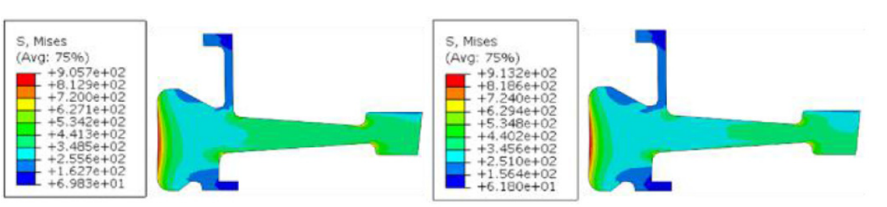

(a) $\mathrm{n}=0.5$

(b) $\mathrm{n}=1.5$

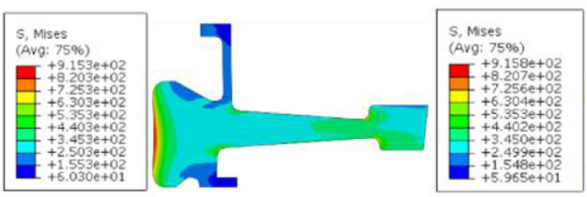

(c) $\mathrm{n}=2.5$

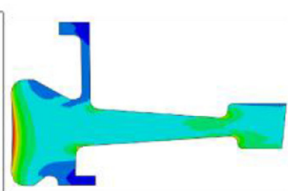

(d) $n=3.5$

Fig. 11. Turbine disk stress distribution diagram with different values of parameter $n$ : (a) $n=0.5$, (b) $n=1.5$, (c) $n=2.5$, and (d) $n=3.5$.

Table 11. Von Mises stress at characteristic nodes of turbine disc for different $n$ values.

\begin{tabular}{llll}
\hline$n$ & $\begin{array}{l}\text { Maximum } \\
\text { von Mises } \\
\text { stress } \\
\text { (MPa) }\end{array}$ & $\begin{array}{l}\text { Von Mises } \\
\text { stress at } \\
\text { minimum } \\
\text { thickness } \\
\text { of turbine } \\
\text { disc (MPa) }\end{array}$ & $\begin{array}{l}\text { Von Mises } \\
\text { stress at } \\
\text { maximum } \\
\text { radius } \\
(\mathrm{MPa})\end{array}$ \\
\hline 0.5 & 905.736 & 421.895 & 367.596 \\
1.0 & 908.268 & 395.180 & 357.907 \\
1.5 & 913.246 & 376.236 & 347.159 \\
2.0 & 914.316 & 369.062 & 342.250 \\
2.5 & 915.296 & 359.385 & 336.005 \\
3.0 & 915.677 & 351.415 & 330.575 \\
3.5 & 915.810 & 344.836 & 325.814 \\
4.0 & 915.859 & 339.394 & 321.606 \\
\hline
\end{tabular}

\section{Effect of FGM composition distribution parameters on performance of turbine discs}

\subsection{Effect of parameter $n$ on stress, strain, and fatigue performance}

We assumed that the engine operated at the maximum state conditions, i.e., at a turbine rotational speed of $17650 \mathrm{rpm}, \mathrm{C} 0=1.0, \mathrm{~K}=1.0$, and $\mathrm{n}=0.5,1,1.5,2.0,2.5$, $3.0,3.5$, and 4.0. Stress analysis calculations were performed for the turbine disc, and the turbine disc stress distribution under different parameter combinations is shown in Figure 11. The von Mises stresses at the characteristic nodes on the turbine disc for different values of $\mathrm{n}$ are shown in Table 11.

The results of Figure 11 and Table 11 show that, as the value of $\mathrm{n}$ increased, the position for maximum von Mises stress was still at the center hole position. The changes in

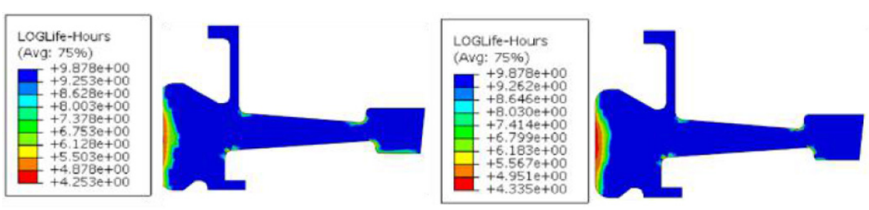

(a) $\mathrm{n}=1.0$

(b) $\mathrm{n}=2.0$

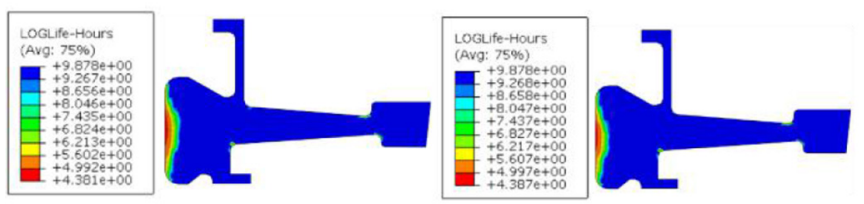

(c) $\mathrm{n}=3.0$

(d) $\mathrm{n}=4.0$

Fig. 12. Simulation results of FGM turbine disc in cruising state: (a) $n=1.0$, (b) $n=2.0$, (c) $n=3.0$, and (d) $n=4.0$.

Table 12. Logarithmic fatigue lives of turbine disc for different $n$ values.

\begin{tabular}{lllll}
\hline$n$ & 1.0 & 2.0 & 3.0 & 4.0 \\
\hline Shortest fatigue life $(\mathrm{h})$ & 17906 & 21627 & 24044 & 24378 \\
Lowest number of cycles & 21345 & 25780 & 28661 & 29059 \\
\hline
\end{tabular}

the maximum von Mises stress amplitude were relatively small, and the high-risk zone of the turbine disk remained the same.

We then investigated the effect of changing the value of $\mathrm{n}$ on the fatigue performance of the turbine disc. The distribution diagrams of the logarithmic fatigue life for $\mathrm{n}$ values of 1.0, 2.0, 3.0, and 4.0 are shown in Figure 12 and the shortest fatigue life of the turbine disc is listed in Table 12. As $\mathrm{n}$ increased, the number of hours of the shortest fatigue life gradually increased while remaining in the range of the low-cycle fatigue life. This occurred because, as the value of $\mathrm{n}$ increased, the material properties of the turbine disc shifted toward that of the GH4169 alloy.

Based on the above analysis results of the stress and fatigue life, the larger the value of $n$ was in the interval $(0,4)$, the better the thermodynamic performance of the FGM turbine disc and the better the low-cycle fatigue performance became.

\subsection{Effect of parameter C0 on stress, strain, and fatigue performance}

Stress analysis calculations were performed for a turbine disc operating under its maximum state conditions, that is, at a rotational speed of $17650 \mathrm{rpm}, \mathrm{n}=2.0, \mathrm{k}=1.0$, and $\mathrm{C} 0=0.1,0.3,0.5,0.7$, and 1.0. The von Mises stress distributions of the turbine disc for various parameter sets are shown in Figure 13. The von Mises stresses at the characteristic nodes of the turbine disc for different values of $\mathrm{C} 0$ are listed in Table 13. As the value of $\mathrm{C} 0$ decreased, the maximum von Mises stress occurred at the same location. The value of the maximum von Mises stress 


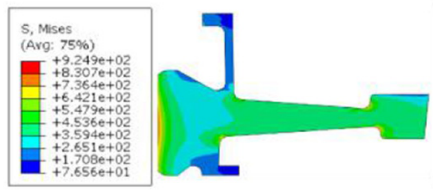

(a) $\mathrm{C} 0=0.1$

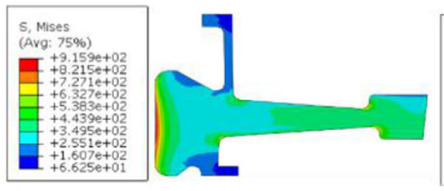

(c) $\mathrm{C} 0=0.7$
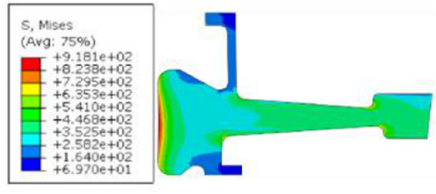

(b) $\mathrm{C} 0=0.5$
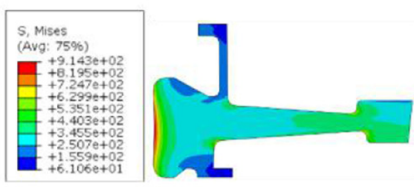

(d) $\mathrm{CO}=1.0$
Fig. 13. Diagram of logarithmic fatigue lives of turbine discs with different values of parameter $n$ : (a) $\mathrm{C} 0=0.1$, (b) $\mathrm{C} 0=0.5$, (c) $\mathrm{C} 0=0.7$, and (d) $\mathrm{C} 0=1.0$.

Table 13. Von Mises stress at characteristic nodes of turbine disc for different $\mathrm{C} 0$ values.

\begin{tabular}{llll}
\hline C0 & $\begin{array}{l}\text { Maximum } \\
\text { von Mises } \\
\text { stress } \\
\text { (MPa) }\end{array}$ & $\begin{array}{l}\text { Von Mises } \\
\text { stress at } \\
\text { minimum } \\
\text { thickness } \\
\text { of turbine } \\
\text { disc (MPa) }\end{array}$ & $\begin{array}{l}\text { Von Mises } \\
\text { stress at } \\
\text { maximum } \\
\text { radius } \\
\text { (MPa) }\end{array}$ \\
\hline 1.0 & 914.316 & 372.098 & 342.250 \\
0.7 & 915.864 & 388.799 & 353.996 \\
0.5 & 918.077 & 401.728 & 361.334 \\
0.3 & 921.125 & 416.686 & 368.319 \\
0.1 & 924.929 & 427.087 & 374.978 \\
\hline
\end{tabular}

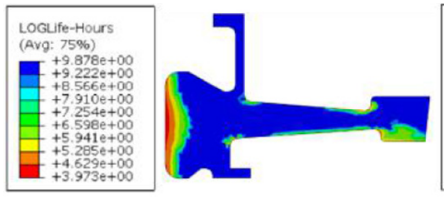

(a) $\mathrm{C} 0=0.1$
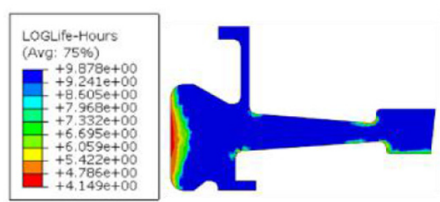

(c) $\mathrm{C} 0=0.7$
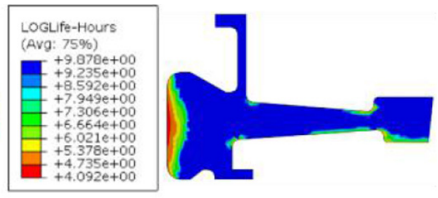

(b) $\mathrm{C} 0=0.5$
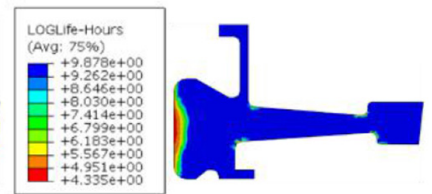

(d) $\mathrm{C} 0=1.0$
Fig. 14. Distribution diagram of the logarithmic fatigue life of the turbine disc with different values of the parameter $\mathrm{C} 0$ : (a) $\mathrm{C} 0=0.1$, (b) $\mathrm{C} 0=0.5$, (c) $\mathrm{C} 0=0.7$, and (d) $\mathrm{C} 0=1.0$.

increased slightly, but the high-risk zone of the turbine disc remained at the center hole.

We then investigated the effect of changing the value of $\mathrm{C} 0$ on the fatigue performance of the turbine disc. The distribution diagrams of the logarithmic fatigue life for different values of $\mathrm{C} 0$ are shown in Figure 14, and the
Table 14. Logarithmic fatigue lives of turbine disc for different $\mathrm{C} 0$ values.

\begin{tabular}{llllll}
\hline C0 & 1.0 & 0.7 & 0.5 & 0.3 & 0.1 \\
\hline $\begin{array}{l}\text { Shortest fatigue } \\
\text { life (h) }\end{array}$ & 21627 & 14093 & 12359 & 10839 & 9397 \\
$\begin{array}{l}\text { Lowest number } \\
\text { of cycles }\end{array}$ & 25780 & 16799 & 14732 & 12920 & 11201 \\
\hline
\end{tabular}

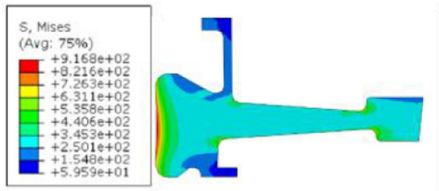

(a) $\mathrm{k}=0.1$

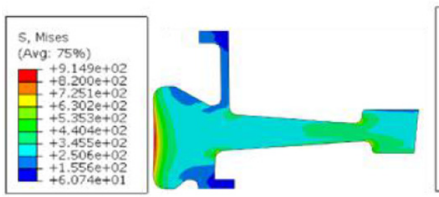

(c) $\mathrm{k}=0.8$

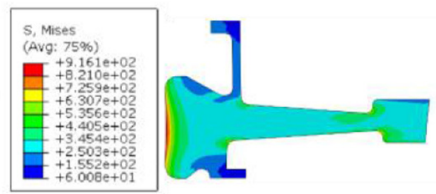

(b) $\mathrm{k}=0.4$

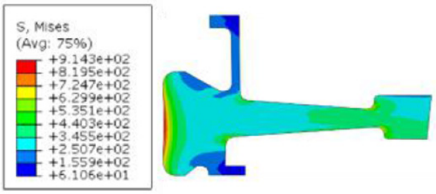

(d) $\mathrm{k}=1.0$
Fig. 15. Turbine disk stress distribution diagram with different values of parameter $\mathrm{k}$ : (a) $\mathrm{k}=0.1$, (b) $\mathrm{k}=0.4$, (c) $\mathrm{k}=0.8$, and (d) $\mathrm{k}=1.0$.

shortest fatigue life of the turbine disc is listed in Table 14. As the value of $\mathrm{C} 0$ decreased, the number of hours of the shortest fatigue life gradually decreased while still remaining in the range of the low-cycle fatigue life. This occurred because, as the value of $\mathrm{C} 0$ decreased, the material properties of the turbine disc shifted toward that of the K418B alloy with lower fatigue strength.Combining the above analysis results of the stress and fatigue life, we learned that the greater the value of $\mathrm{C} 0$ was in the range of $(0,1)$, the better the thermodynamic performance of the FGM turbine disc and the better the low-cycle fatigue performance became.

\subsection{Effect of parameter $k$ on stress, strain, and fatigue performance}

The von Mises stress distributions shown in Figure 15 were obtained for different parameter sets with the turbine disc operating at the maximum state condition, that is, at a rotational speed of $17650 \mathrm{rpm}, n=2.0, \mathrm{C} 0=1.0$, and $\mathrm{k}=0.1,0.2,0.4,0.6,0.8$, and 1.0. The turbine disc von Mises stress distribution under different parameter combinations is shown in Figure 15. The values of the von Mises stress at the characteristic nodes on the turbine disc are listed in Table 15.

The table shows that, as the value of $\mathrm{k}$ decreased, the position for the maximum von Mises stress remained unchanged on the turbine disc. The magnitudes of the changes of the maximum von Mises stress were relatively 
Table 15. Von Mises stress at characteristic nodes of turbine disc for different $\mathrm{k}$ values.

\begin{tabular}{llll}
\hline $\mathrm{k}$ & $\begin{array}{l}\text { Maximum } \\
\text { von Mises } \\
\text { stress (MPa) }\end{array}$ & $\begin{array}{l}\text { Von Mises } \\
\text { stress at } \\
\text { minimum } \\
\text { thickness } \\
\text { of turbine } \\
\text { disc (MPa) }\end{array}$ & $\begin{array}{l}\text { Von Mises } \\
\text { stress at } \\
\text { maximum } \\
\text { radius } \\
\text { (MPa) }\end{array}$ \\
\hline 1.0 & 914.316 & 372.098 & 342.250 \\
0.8 & 914.934 & 365.108 & 329.117 \\
0.6 & 915.518 & 353.963 & 316.134 \\
0.4 & 916.070 & 335.213 & 303.304 \\
0.2 & 916.587 & 323.909 & 290.630 \\
0.1 & 916.832 & 318.256 & 284.353 \\
\hline
\end{tabular}

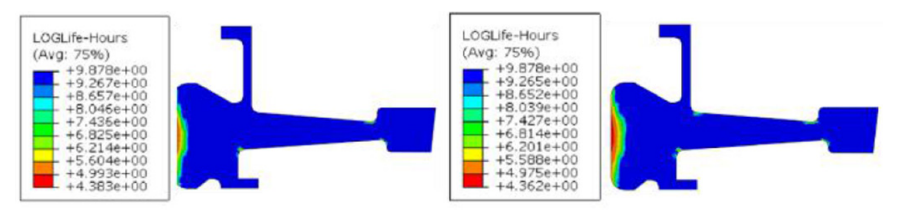

(a) $\mathrm{k}=0.2$

(b) $\mathrm{k}=0.4$

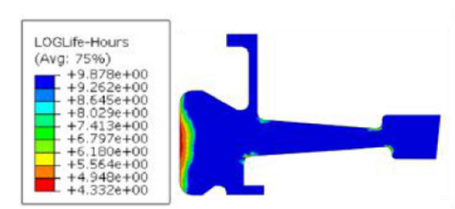

(c) $\mathrm{k}=0.6$
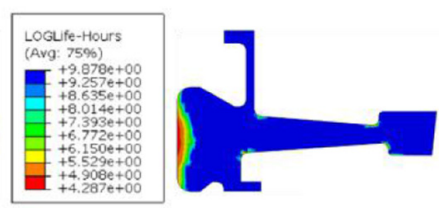

(d) $\mathrm{k}=0.8$
Fig. 16. Distribution diagram of logarithmic fatigue life of turbine disk with different values of parameter $\mathrm{k}$ : (a) $\mathrm{k}=0.2$, (b) $\mathrm{k}=0.4$, (c) $\mathrm{k}=0.6$, and (d) $\mathrm{k}=0.8$.

small, and the center of the disk remained in the danger zone for disc failure. We then investigated the effect of changing the value of $\mathrm{k}$ on the fatigue performance of the turbine disc. The logarithmic fatigue life distributions for $\mathrm{k}$ values of $0.2,0.4,0.6$, and 0.8 are shown in Figure 16, and the shortest fatigue life of the turbine disc is listed in Table 16. As $\mathrm{k}$ increased, the number of hours of the shortest fatigue life gradually increased while remaining in the range of the low-cycle fatigue life.

Based on the above analysis results of the stress and fatigue life, we determined that the smaller the value of $\mathrm{k}$ was in the interval $(0,1)$, the better the thermodynamic performance of the FGM turbine disc and the better the low-cycle fatigue performance became.

\section{Conclusion}

In this work, we investigated a bimetallic functionally graded material turbine disc and successfully modelled an FGM turbine disc by combining theoretical analysis and finite element numerical simulations. The thermodynamic
Table 16. Logarithmic fatigue lives of turbine disc for different $\mathrm{k}$ values.

\begin{tabular}{lllll}
\hline $\mathrm{k}$ & 0.8 & 0.6 & 0.4 & 0.2 \\
\hline Shortest fatigue life $(\mathrm{h})$ & 19364 & 21478 & 23014 & 24154 \\
Lowest number of cycles & 23082 & 25603 & 27434 & 28792 \\
\hline
\end{tabular}

responses of an FGM turbine disc under service conditions were obtained based on finite element simulations. Lowcycle fatigue calculations of the turbine disc were then performed. Finally, the correlation between the composition distribution parameters and the service performance of the FGM turbine disc was obtained by repeatedly calculating the thermodynamic responses and low-cycle fatigue results while adjusting the FGM composition distribution parameters. The main conclusions were as follows:

- By combining the existing theoretical models of functionally graded materials and mechanical calculation methods, we proposed a volumetric fraction expression and adjustable composition distribution parameters to simulate the composition distribution of turbine discs applicable for aero-engines. A thermodynamic response solution based on the finite element method was found for FGM turbine discs.

- The distributions of the stress, strain, and total deformation of an FGM turbine disc under various working conditions were obtained. The results showed that, for an FGM turbine disc under centrifugal and thermal loads, the stress gradually decreased from the center hole to the rim, the maximum stress appeared at the center hole of the disc, and the total deformation appeared at the rim. The research results verified that the high-risk position of the FGM disc and the GH4169 alloy disc during service were both in the center hole of the turbine disk.

- The distributions of the fatigue damage, FOS factor, and fatigue life of the FGM turbine disc operating in the maximum state were calculated. Based on the simulation results, the maximum damage, minimum FOS factor, and shortest fatigue life of the FGM disc appeared at the high-risk position on the disc, and the fatigue life met the criterion of the low-cycle fatigue life range.

- Based on the volumetric fraction expression of the simulated functionally graded material composition distribution, the influences of the adjustable values of the composition distribution parameters in the expression of the FGM disc on the service performance were studied. The results showed that the greater the value of parameter $\mathrm{n}$ was in the $(0,4)$ range, the greater the value of $\mathrm{C} 0$ was in the $(0,1)$ range. The smaller the value of $\mathrm{k}$ was in the $(0,1)$ range, the better the thermodynamic performance of the FGM turbine disc and the better the low-cycle fatigue performance became.

Acknowledgements. This study was financially supported by the National Natural Science Foundation of China (grant number 51705440), the Fundamental Research Funds for the Central 
Universities XMU (grant number 20720180072), the Aeronautical Science Foundation of China (grant number 20170368001), the Shenzhen Fundamental Research Program (grant number JCYJ20170818141303656), and the Natural Science Foundation of Fujian Province, China (grant number 2019J01044).

\section{References}

[1] J. Rahimi, E. Poursaeidi, E. Khavasi, Stress analysis of a second stage gas turbine bladeunderasymmetricthermal gradient, Mechanics \& Industry 20, 607 (2019)

[2] V. Birman, L.W. Byrd, Modeling and analysis of functionally graded materials and structures, Applied Mechanics Reviews 60, 195 (2007)

[3] Z. Sun, J.C. Ion, Review laser welding of dissimilar metal combinations, Journal of Materials Science 30, 4025-4214 (1995)

[4] Y.J. Xu, P. Zhang, W.H. Zhang, Two-scale micromechanical modeling of the time dependent relaxation modulus of plain weave polymer matrix composites, Composite Structures 123, 35-44 (2015)

[5] X.Y. Hui, Y.J. Xu, W.H. Zhang, Multi-scale modeling of micro curing residual stresses evolution in carbon fiber reinforced thermoset polymer composites, Frontiers of Mechanical Engineering 15, 475-483 (2020)

[6] B.E. Carroll, R.A. Otis, J.P. Borgonia, J. Suh, R.P. Dillon, A.A. Shapiro, A.M. Beese, Functionally graded material of 304L stainless steel and inconel 625 fabricated by directed energy deposition: characterization and thermodynamic modeling, Acta Materialia 108, 46-54 (2006)

[7] R.M. Mahamood, E.T. Akinlabi, Types of functionally graded materials and their areas of application, in: Functionally Graded Materials. (Springer, Cham, 2017, pp. 9-21)

[8] S. Pandey, S. Pradyumna, Transient stress analysis of sandwich plate and shell panels with functionally graded material core under thermal shock, Journal of Thermal Stresses 41, 534-567 (2018)

[9] H. Parandvar, M. Farid, Nonlinear dynamic response of functionally graded shallow shells under harmonic excitation in thermal environment using finite element method, Composite Structures 149, 351-361 (2016)

[10] M. Jabbari, S. Sohrabpour, M.R. Eslami, Mechanical and thermal stresses in a functionally graded hollow cylinder due to radially symmetric loads, International Journal of Pressure Vessels and Piping 79, 493-497 (2002)

[11] Z.S. Shao, Mechanical and thermal stresses of a functionally graded circular hollow cylinder with finite length, International Journal of Pressure Vessels and Piping 82, 155-163 (2005)

[12] J.N. Reddy, Analysis of functionally graded plates, International Journal for Numerical Methods in Engineering 47, 663-684 (2000)

[13] R. Pourhamid, M.T. Ahmadian, H.M. Moghaddam et al., Mechanical analysis of a functionally graded cylinder-piston under internal pressure due to a combustion engine using a cylindrical super element and considering thermal loading, Scientia Iranica 22, 493-503 (2015)

[14] S. Zhang, D. Zhao (Eds.) Aerospace materials handbook, CrCPress, Boca Raton, FL, USA, 2012

[15] Z.S. Shao, Mechanical and thermal stresses of a functionally graded circular hollow cylinder with finite length, International Journal of Pressure Vessels and Piping 82, 155-163 (2004)

[16] L. Wu, Z. Jiang, J. Liu, Thermo elasticstability of functionally graded cylindrical shells, Composite Structures 70, 60-68 (2004)

[17] G.R. Johnson, W.H. Cook, A constitutive model and data for metals subjected to large strains, high strain rates and high temperatures, in: Proceedings of the 7th International Symposium on Ballistics, Den Haag, The Netherlands, 1983, 21, pp. 541-547

[18] P. Geng, G. Qin, J. Zhou et al., Hot deformation behavior and constitutive model of GH4169 superalloy for linear friction welding process, Journal of Manufacturing Processes 32, 469-481 (2018)

[19] L. Witek, Failure analysis of turbine disc of an aero engine, Engineering Failure Analysis 13, 9-17 (2006)

[20] Z.Y. Xue, X.A. Hu, G.F. Rao, Thermal-mechanical coupling and center hole fatigue crack growth of turbine disks, . Journal of Nanchang Hangkong University (Natural Sciences) 33, 39-49 (2019) (in Chinese)

[21] S.P. Zhu, Q. Liu, Q. Lei et al., Probabilistic fatigue life prediction and reliability assessment of a high pressure turbine disc considering load variations, International Journal of Damage Mechanics 27, 1569-1588 (2018)

[22] A.X. Ding, J. Wu, Spey MK202 engine stress criteria (EGD-3), (International Aviation Editorial Dept., Beijing, China, 1979)

Cite this article as: Y. Dong, W. Yan, T. Liao, Q. Ye, Y. You, Model characterization and mechanical property analysis of bimetallic functionally graded turbine discs, Mechanics \& Industry 22, 4 (2021) 